\title{
Very low velocity ion slowing down in binary ionic mixtures: Charge- and mass-asymmetry effects
}

\author{
Patrice Fromy \\ Direction de l'Informatique, Université Paris XI, 91405 Orsay, France \\ Bekbolat Tashev \\ Department of Physics, Kazakh National University, Almaty 480012, Kazakhstan \\ Claude Deutsch \\ LPGP (UMR-CNRS 8578), Université Paris XI, 91405 Orsay, France \\ (Received 5 May 2010; published 1 October 2010; corrected 15 October 2010)
}

\begin{abstract}
A binary ionic mixture (BIM) in dense and hot plasmas of specific concern for inertial confinement fusion and white dwarf crust is considered as a target for incoming light ions with a velocity smaller than the thermal electron one. The given target stopping power, mostly BIM monitored, is specifically studied in terms of charge and mass asymmetry in its ionic component. The classical plasma target is worked out within a dielectric framework, and scanned with respect to density, temperature, and BIM composition.
\end{abstract}

\section{INTRODUCTION}

Low velocity ion slowing down (LVISD) has been for a long time a very important topic featuring the last stage of the ion-dense target interaction. Initially, the given targets were taken as cold ones, with projectile ion velocity $V_{p}$ currently contrasted to orbital velocities of electrons bound to that target [1]. Here we address the LVISD issues in fully ionized plasmas built on binary ionic mixtures (BIM) such as the deuterium-tritium (DT) BIM of current interest in thermonuclear fusion research. In particular, the control of the resulting alpha particles, is of paramount significance in the conduct of inertial fusion energy (IFE) as well as magnetic fusion energy (MFE) senarii to economical success. The given control demands a basic and fundamental understanding of the $\alpha$ 's LVISD, which, produced at $3.5 \mathrm{MeV}$, lose most of their energy to the surrounding plasma with a $V_{p} \leq V_{\text {the }}$ electron thermal velocity. Similar considerations apply to the present U.S. program devoted to the production of warm dense matter with low velocity ions linearly accelerated, and fully stopped in complex and initially cold targets [2]. Here, the accelerator parameters are tuned so that the largest ion projectile energy loss takes in the vicinity of the Bragg peak, near the end of range [3]. In this latter case, the goal is to produce plasmas with a density between $1 / 10$ and 10 times solid density, with a temperature below $10 \mathrm{eV}$, in order to probe the whole scale of strongly coupled ions and arbitrary degeneracy in the neutralizing electron fluid.

Considering the BIM target with neutralizing electrons, in a dielectric framework, the ion beam-target interaction is essentially qualified through a dimensionless ratio of average collision parameter $\langle b o\rangle$ and dynamical screening length, $\lambda$, given as [4]

$$
\frac{\langle b o\rangle}{\lambda}=\frac{\left|Z_{\mathrm{eft}}\right|}{N_{D}} \cdot \frac{1}{3\left(1+\frac{V_{p}^{2}}{V_{\text {the }}^{2}}\right)^{3 / 2}},
$$

maximized at $V_{p}=0$ with $N_{D}=n_{e} \lambda_{D}^{3}$, in terms of target electron density $n_{e}$ and Debye length $\lambda_{D}(\mathrm{~cm})=$ $743 \sqrt{\frac{T(\mathrm{eV})}{n_{e}\left(\mathrm{~cm}^{-3}\right)}}$.

In the sequel, we shall restrict to weakly coupled plasma targets with $N_{D} \gg 1$, which nonetheless encompass most plasmas of IFE and MFE interest. We shall work out LVISD with $V_{p}$ alternatively scaled to $V_{\text {the }}$ and $V_{\text {thi }}$, ion thermal velocity, in order to probe its very low $V_{p}$ specific features, arising mostly from Coulomb interaction between incoming ion projectile and target BIM.

The given ion beams are taken dynamically uncorrelated.

The remaining part of this work is structured as follows. Section II deals with LVISD technicalities considered within dielectric formalism grounded on the Fried-Conte [5] classical formulation used additively for the neutralizing electrons and BIM, as well.

Then, we develop a parameter study based on increasing values of charge $Z_{2} / Z_{1}$ and mass $M_{2} / M_{1}$ ratios for the target BIM built on pointlike classical ions $\left(Z_{1}, M_{1}\right)$ and $\left(Z_{2}, M_{2}\right)$. Section III is thus first devoted to the nearly symmetric DT fuel. Section IV details asymmetries introduced by arbitrary $M_{2} / M_{1}$ at fixes $Z_{2} / Z_{1}$. From Sec. V, we investigate several BIM with $Z_{2} / Z_{1}>1$, of considerable interest for thermonuclear fusion and astrophysics as well. The specificities of neutral BIM including antiprotons (pbars) are briefly sketched in Sec. VI. Outlooks are offered in Sec. VII. 


\section{DIELECTRIC STOPPING FORMALISM}

We modelize dense and mostly hot plasma targets as binary ionic mixtures (BIM) of pointlike and classical ions $\left(M_{1}, Z_{1}\right)$ and $\left(M_{2}, Z_{2}\right)$, neutralized by a classical electron fluid described within the framework of the Fried-Conte dielectric function [5].

In such an approach, it appears useful to work with the overall dielectric function

$\varepsilon(\vec{k}, \omega)=1+\frac{1}{k^{2}}\left[W\left(\frac{\omega}{k}\right)+W\left(\sqrt{M_{1}} \frac{\omega}{k}\right)+W\left(\sqrt{M_{2}} \frac{\omega}{k}\right)\right]$

with the usual Fried-Conte dispersion function $W(\operatorname{Im} \zeta \geq$ 0)

$$
W(\mathrm{~s})=\frac{1}{\sqrt{2 \pi}} \lim _{\nu \rightarrow 0+} \int_{-\infty}^{\infty} d x \frac{x e^{-x^{2} / 2}}{x-\mathrm{s}-i \nu}
$$

and $X(\zeta)=\operatorname{Re} W(\zeta), Y(\zeta)=\operatorname{Im} W(\zeta)$. Generalizing linearly through expression (2), the standard one-component stopping quadrature $[6,7]$

$$
\begin{aligned}
-\left[\frac{d E}{d x}\right]= & \frac{Z^{2} N_{D}}{(2 \pi)^{2}} \int_{0}^{k_{\max }} d k k^{3} \int_{-1}^{+1} d \mu \\
& \times \frac{\mu Y\left(\mu V_{p}\right)}{\left[k^{2}+X\left(\mu V_{p}\right)\right]^{2}+Y^{2}\left(\mu V_{p}\right)}
\end{aligned}
$$

with $Z=Z_{\text {eff }} / N_{D}$, where $Z_{\text {eff }}$ denotes the projectile effective charge at velocity $v_{p}, N_{D}=n_{e} \lambda_{\mathrm{De}}^{3}$ in terms of target electron density and corresponding Debye length. In the sequel, $v_{p}$ will be scaled by $v_{\text {the }}=\sqrt{k_{B} T / m_{e}}$, thermal electron velocity with $T$, thermalized target temperature. In Eq. (3), we have to pay attention to the selection of maximum cutoff $k_{\max }$, taking into account quantum effects diffraction $\left(k_{B} T \geq 1 \mathrm{Ry}\right)$ in a high temperature plasma. So, one explicits $[6,7]$

$$
\begin{aligned}
k_{\mathrm{max}} & =\min \left(\frac{m\left(V_{p}^{2}+V_{\mathrm{the}}^{2}\right.}{Z_{\mathrm{eff}} e^{2}}, \frac{2 m \sqrt{V_{p}^{2}+V_{\mathrm{the}}^{2}}}{\hbar}\right) \\
& =\min \left(\frac{4 \pi}{Z}\left(v_{p}^{2}+1\right), 8 \pi \sqrt{2} N_{D} \frac{\alpha c}{V_{\mathrm{the}}} \sqrt{v_{p}^{2}+1}\right),
\end{aligned}
$$

where $v_{p}$ is now dimensionless on the second line on the right-hand side. $\alpha=1 / 137$ is the fine structure constant, and $c$ is light velocity. In this regard, it should be noticed that the occurrence of quantum diffraction $k_{\max }$ is restricted to the electron fluid component of the target. The corresponding BIM will always appear as pointlike classical, as long as one restricts to the velocity range $V_{\text {thi }} \leq V_{p} \leq V_{\text {the }}$. In adapting Eq. (3) to BIM stopping, it proves convenient to introduce the relative ion concentration of species 1, i.e.,

$$
\alpha=\frac{N_{1}}{N_{1}+N_{2}},
$$

in terms of ion number $N_{i}$ with $i=1,2$, in target plasma, so that BIM densities,

$n_{1}=\frac{n_{e} \alpha}{\bar{Z}}, \quad n_{2}=\frac{n_{e}(1-\alpha)}{\bar{Z}}, \quad \bar{Z}=Z_{1} \alpha+Z_{2}(1-\alpha)$,

are straightforwardly expressed in terms of electron density $n_{e}$.

Then, we can estimate the stopping contributions of every target component: electron (0), ion 1 , and ion 2 as follows:

$$
\begin{aligned}
& -\frac{d E_{0}}{d x}=C_{0} \int_{0}^{k_{\max _{0}}} d k k^{3} \int_{-1}^{+1} d \mu \frac{\mu Y\left[\mu v_{p}\right]}{D\left[k, \mu v_{p}\right]}, \\
& -\frac{d E_{1}}{d x}=C_{1} \int_{0}^{k_{\max _{1}}} d k k^{3} \int_{-1}^{+1} d \mu \frac{\mu Y\left[\sqrt{M_{1} \mu v_{p}}\right]}{D\left[k, \mu v_{p}\right]}, \\
& -\frac{d E_{2}}{d x}=C_{2} \int_{0}^{k_{\max _{2}}} d k k^{3} \int_{-1}^{+1} d \mu \frac{\mu Y\left[\sqrt{M_{2} \mu v_{p}}\right]}{D\left[k, \mu v_{p}\right]},
\end{aligned}
$$

where

$$
\begin{aligned}
D\left[k, \mu v_{p}\right]= & \left(k^{2}+X\left[\mu_{p} v_{p}\right]+X\left[\sqrt{M_{1}} \mu v_{p}\right]\right. \\
& \left.+X\left[\sqrt{M_{2}} \mu v_{p}\right]\right)^{2}+\left(Y\left[\mu v_{p}\right]\right. \\
& \left.+Y\left[\sqrt{M_{1}} \mu v_{p}\right]+Y\left[\sqrt{M_{2}} \mu v_{p}\right]\right)^{2}, \\
C_{0} & =\frac{k_{B} T Z_{\text {eff }}^{2}}{4 \pi^{2} n_{e} \lambda_{D}^{4}} \\
C_{1} & =\frac{C_{0} Z_{1}^{4} \alpha}{\bar{Z}} \\
C_{2} & =\frac{C_{0} Z_{2}^{4}(1-\alpha)}{\bar{Z}}
\end{aligned}
$$

altogether with

$$
\begin{aligned}
k_{\max _{0}}= & \min \left(k_{\max _{0}^{1}}=n_{e} \lambda_{D}^{3} \frac{4 \pi\left(v_{p}^{2}+1\right)}{Z_{\mathrm{eff}}},\right. \\
& \left.185.73 n_{e} \lambda_{D}^{3} \sqrt{\frac{v_{p}^{2}+1}{T(\mathrm{eV})}}\right), \\
k_{\max _{1}}= & \frac{k_{\max _{0}^{1}}}{Z_{1}^{3}}\left(\frac{\bar{Z}}{\alpha}\right)^{1 / 2}, \\
k_{\max _{2}}= & \frac{k_{\max _{0}^{1}}}{Z_{2}^{3}}\left(\frac{\bar{Z}}{1-\alpha}\right)^{1 / 2} .
\end{aligned}
$$

Expressions (7a)-(7c) are in $k_{\mathrm{BT}} / \lambda_{D}$, with the Debye screening length $\lambda_{D}$, respectively, adapted to electron, ion 1 , and ion 2. $V_{p}$ is in $V_{\text {the }}$. 

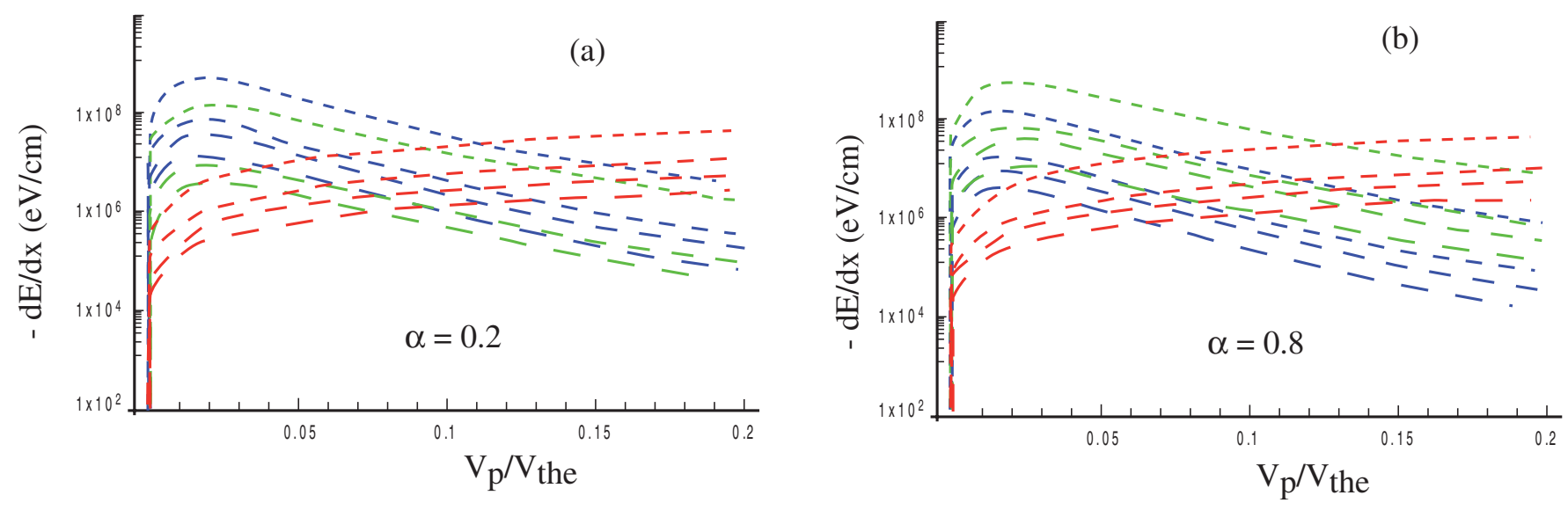

FIG. 1. (Color) Proton particle projectile in a deuterium-tritium BIM with $n_{e}=10^{23} \mathrm{e}-\mathrm{cm}^{-3}$ and temperatures $=(\mathbf{- ~ - ~ - ~ - ~ - ~ - ~ ) ~} 100 \mathrm{eV}$, ( - - - ) $1 \mathrm{keV},\left(\right.$ - - - ) $2 \mathrm{keV}$, and ( - - - ) $5 \mathrm{keV}$ with $D^{+}$relative proportion: (a) $\alpha=0.2$ and (b) $\alpha=0.8$. Dark gray (red) refers to electron stopping, light gray (green) to $D^{+}$stopping, and black (blue) denotes $T^{+}$stopping, $-d E / d x(\mathrm{eV} / \mathrm{cm}$ ) in terms of $V_{p} / V_{\text {the }}$.

The target electron cutoff estimate (10a) shows that corresponding $\hbar \neq 0$ corrections should be included for equilibrium temperatures $T=T_{e}=T_{i} \geq 110 \mathrm{eV}$. Target ion slowing down contributions remain classical as long as $T \leq 200\left(M_{i} / 1836\right) \mathrm{keV}$, provided the right-hand side of Eqs. (10b) and (10c) replaces $v_{p}^{2}+1$ by $M_{1} v_{p}^{2}+1$ and $M_{2} v_{p}^{2}+1$, respectively, with $V_{p}$ in $V_{\text {the }}$. When target ions enter the $\hbar \neq 0$ regime, the right-hand term within brackets in Eq. (10a) becomes $7930 \sqrt{\left(v_{p}^{2}+1\right)} \frac{M_{i}}{1836 T(\mathrm{eV})}$ for ion species $i$.

For $k_{\max } \gg 1$, it appears convenient to extend a previous analytic LVISD expression [6], to the present BIM situation with

$$
-\frac{d E}{d x}=\frac{Z^{2} N_{D}}{12 \pi \sqrt{2 \pi}} \frac{V_{p}}{V_{\text {the }}} \sqrt{\bar{M}} \ell n\left[\bar{M}^{2} \frac{32 \pi^{2}}{Z 2}\right]
$$

where $Z=Z_{\text {eff }} /\left(n_{e} \lambda_{D}^{3}\right)$ and $\bar{M}=\alpha M_{1}+(1-\alpha) M_{2}$

\section{QUASISYMMETRIC BIM}

We begin our analysis with the quasisymmetric BIM $D^{+}-T^{+}$, of paramount significance in present thermonuclear fusion research [8]. Figure 1 features rather convincingly the expected quasisymmetric behavior $\alpha \leftrightarrow 1-\alpha$, for the proton projectiles low ion velocity slowing down, for a rather dense plasma target of inertial fusion energy (IFE) concern [8] with $n_{e}=10^{23} \mathrm{e}-\mathrm{cm}^{-3}$ and a rather wide temperature range $T(\mathrm{eV}) \varepsilon[10,5000]$. Stopping data $-d E / d x$ are given in $\frac{T(\mathrm{eV})}{\lambda_{D}(\mathrm{~cm})}$, with $\lambda_{D}(\mathrm{~cm})=743 \sqrt{\frac{T(\mathrm{eV})}{n_{e}\left(\mathrm{~cm}^{-3}\right)}}$, target electron Debye length, in terms of $V_{p} / V_{\text {the }}$. BIM stopping contributions, light gray (green) for $D^{+}$stopping, and black (blue) for $T^{+}$stopping, display mostly a negative
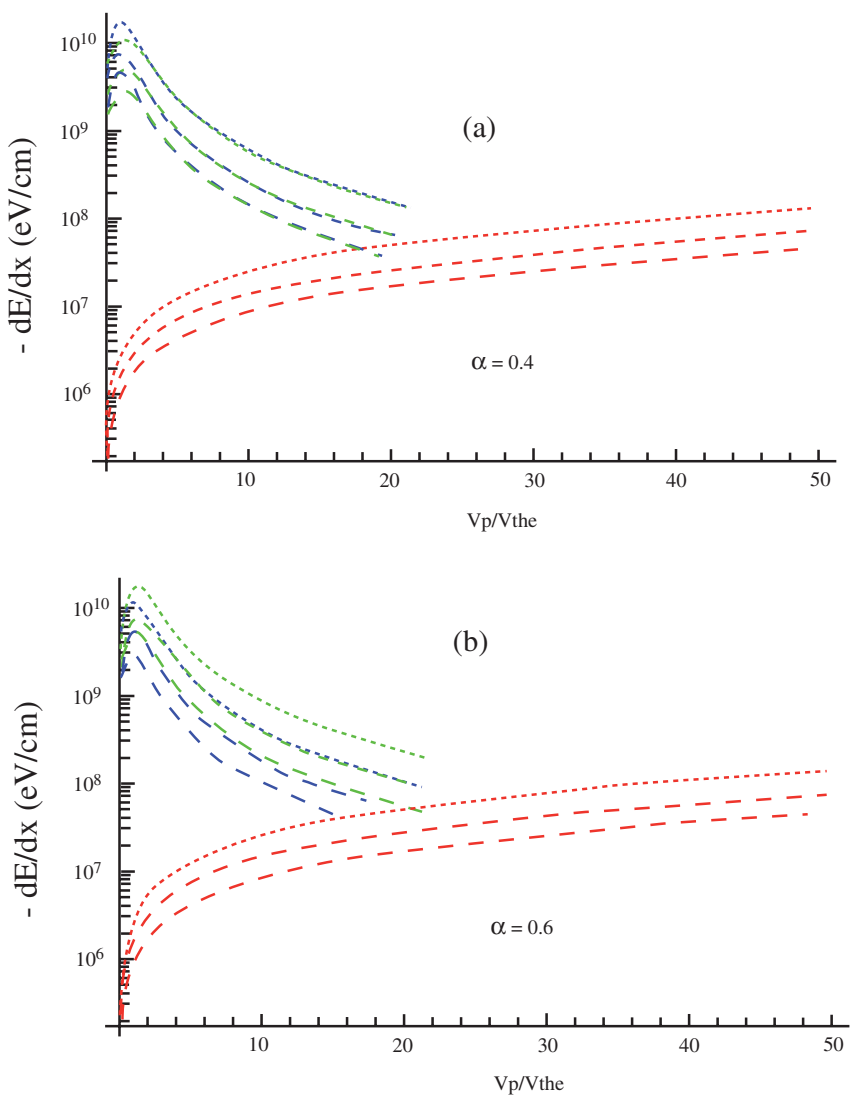

FIG. 2. (Color) Proton particle projectile in a deuterium-tritium BIM with $n_{e}=10^{26} \mathrm{ecm}^{-3}$ and temperatures $=(\ldots . . .$. $1 \mathrm{keV},\left(\right.$ - - - - ) $2 \mathrm{keV},\left(\right.$ - - ) $10 \mathrm{keV}$ with $D^{+}$relative proportion: (a) $\alpha=0.4$ and (b) $\alpha=0.6$. Dark gray (red) refers to electron stopping, light gray (green) to $D^{+}$stopping, and black (blue) denotes $T^{+}$stopping, $-d E / d x(\mathrm{eV} / \mathrm{cm})$ in terms of $V_{p} / V_{\text {the }}$. 

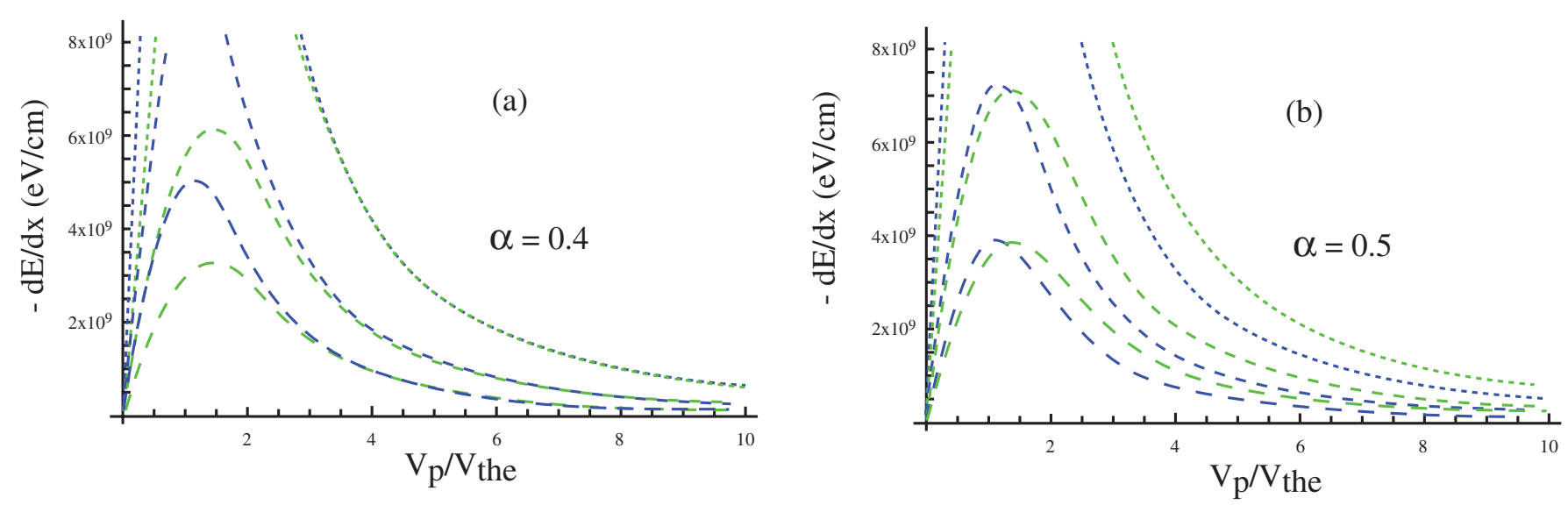

FIG. 3. (Color) Proton particle projectile in a deuterium-tritium BIM with $n_{e}=10^{26} \mathrm{e} \mathrm{cm}^{-3}$ and temperatures $=(\cdots \cdots \cdots) 1 \mathrm{keV}$, ( - - - ) $2 \mathrm{keV},\left(\right.$ - $_{-}$-) $10 \mathrm{keV}$, with $D^{+}$relative proportion: (a) $\alpha=0.4$ and (b) $\alpha=0.5$. Dark gray (red) refers to electron stopping, light gray (green) to $D^{+}$stopping, and black (blue) denotes $T^{+}$stopping, $-d E / d x(\mathrm{eV} / \mathrm{cm})$ in terms of $V_{p} / V_{\text {the }}$.

slope, and cross the dark gray (red) electron-stopping contribution for $0.05 \leq V_{p} / V_{\text {the }} \leq 0.2$, with an increasing one.

It should also be appreciated that the least abundant $i$ component (smallest $\alpha$ ) crosses the $e$ contribution at lower $V_{p}$ values.

Best matching between $D^{+}$-and $T^{+}$—curves occurs around $\alpha=0.4$, for $V_{p} / V_{\text {the }} \geq 0.1$. At $\alpha=0.5$, the lightest ion $D^{+}$provides a stopping contribution (SC) slightly above the $T^{+}$one, in log scale, due to the modest $M_{D^{+}}-M_{T^{+}}$mass difference. Switching the above exploration to the core plasma envisioned in the fast ignition scenario for IFE, one witnesses the pertaining LVISD quantities in Fig. 2 for $n_{e}=10^{26} \mathrm{e}-\mathrm{cm}^{-3}$ with $T(\mathrm{keV})=$ 2 , 5, and 10 , in terms of $V_{\text {th } 1}=V_{\text {the }} / 60$, on a range equivalent to that of Fig. 1.

Again an excellent agreement between $D^{+}$-and $T^{+}$stopping is observed at $V_{p} / V_{\text {thi }} \geq 1$, i.e. $=V_{p} / V_{\text {the }} \geq$ 0.016 (Fig. 1).

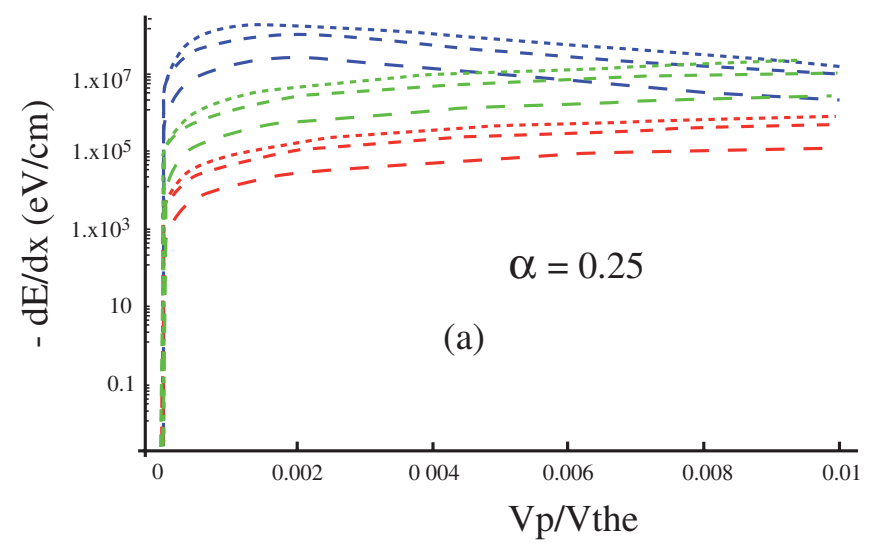

Figures 1 and 2 basically feature a linear in $V_{p} \ll V_{\text {the }} e$ stopping, contrasted to strongly asymmetric bell-shaped $i$-stopping curves, with a Bethe-like decay $\sim V_{p}^{-2}$ for $V_{p} \geq$ $V_{\text {thi }}$. The given bell shapes turn much more symmetrical with $-d E / d x$ in linear scale (Fig. 3), on a reduced $V_{p} / V_{\text {thi }} \leq 10$ range. Then, the $e$ stopping becomes hardly noticeable, on the $V_{p}$ axis. The optimized matching at $\alpha=$ 0.4 between $D^{+}$—and $T^{+}$— stopping is also very accurately documented.

\section{MASS ASYMMETRY $\left(Z_{1}=Z_{2}=1, M_{2} / M_{1} \gg 1\right)$}

The above $D^{+}-T^{+}$captions (Figs. 1-3) dedicated to BIM with $M_{1} / M_{2}=2 / 3$ feature parallel stopping contributions with no crossing, except with the electron one. Here, we enlarge considerably the $M_{2} / M_{1}$ ratio while keeping same charge unity on $\mathrm{H}^{+}$and $\mathrm{U}_{238}^{+}$(Figs. 4 and 5 ), in dense IFE plasmas with $n_{e}=10^{23} \mathrm{e}-\mathrm{cm}^{-3}$ and $T(\mathrm{keV})=1,5$, and 10 , submitted to proton projectiles.

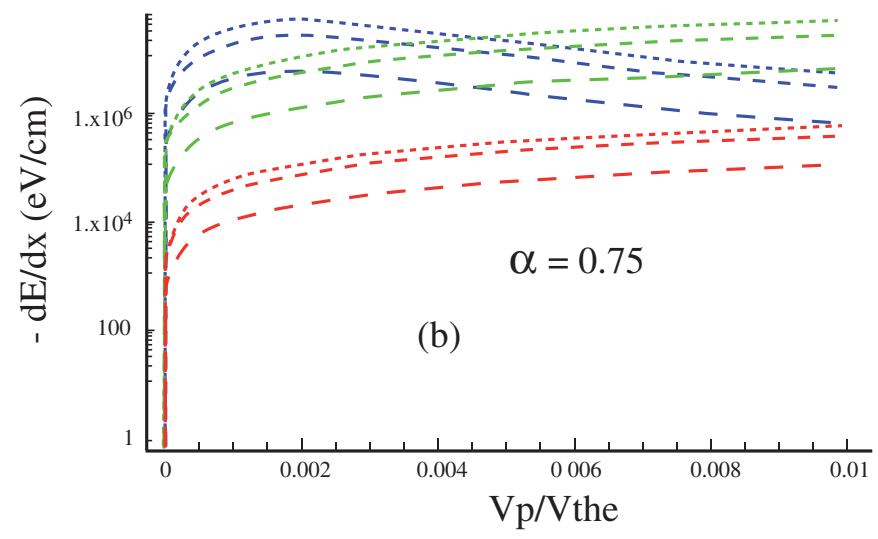

FIG. 4. (Color) Proton particle projectile in a proton- $\mathrm{U}_{238}^{+} \mathrm{BIM}$ with $n_{e}=10^{26} \mathrm{e} \mathrm{cm}^{-3}$ and temperatures $=(\ldots . . . .) .1 \mathrm{keV}$, ( - - - - ) $5 \mathrm{keV},\left(-\right.$ - $\left.^{-}\right) 10 \mathrm{keV}$, with $\mathrm{H}^{+}$relative proportion: (a) $\alpha=0.25$, (b) $\alpha=0.75$. Dark gray (red) refers to electron stopping, light gray (green) to $D^{+}$stopping, and black (blue) denotes $T^{+}$stopping, $-d E / d x(\mathrm{eV} / \mathrm{cm})$ in terms of $V_{p} / V_{\text {the }}$. 

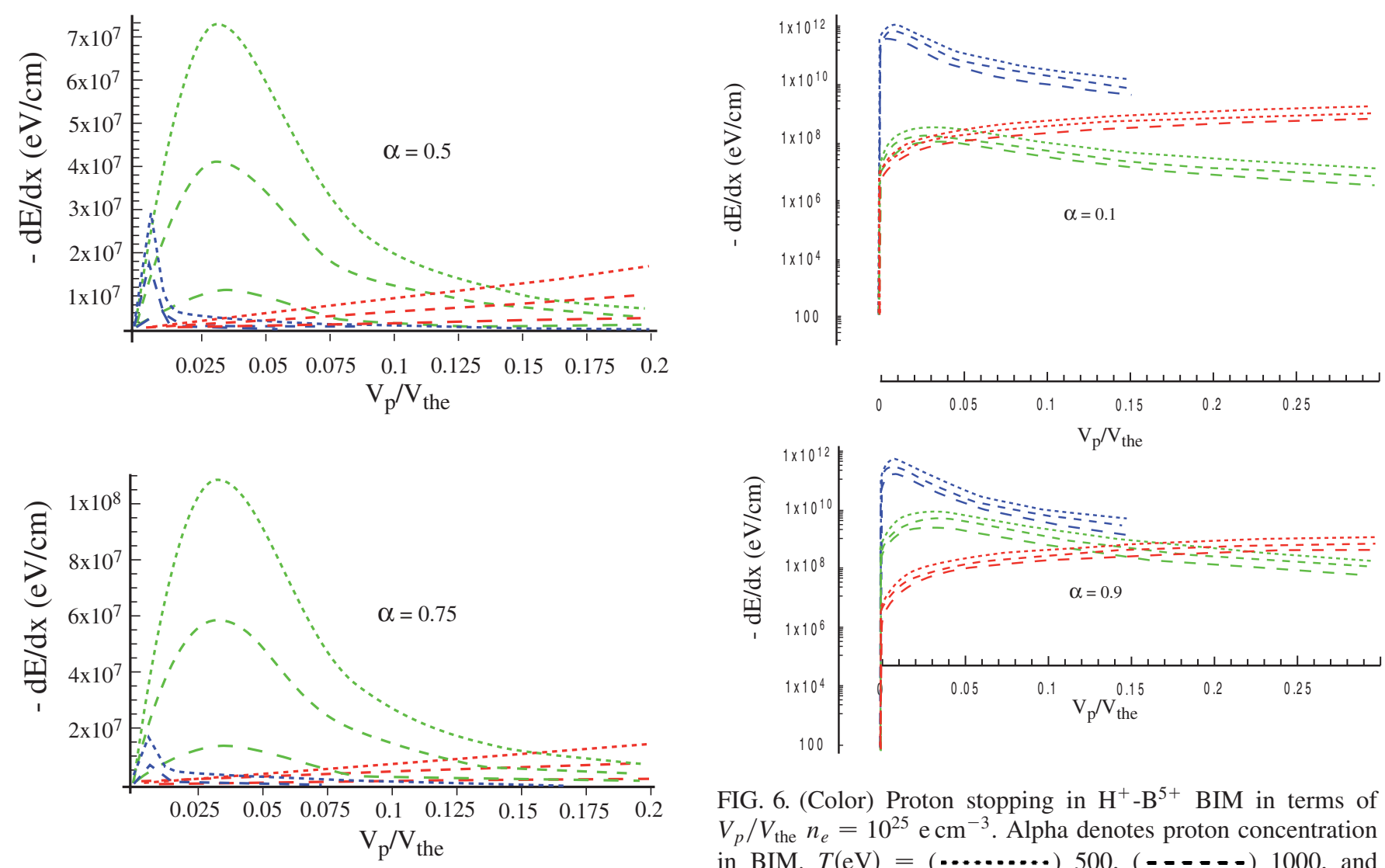

FIG. 6. (Color) Proton stopping in $\mathrm{H}^{+}-\mathrm{B}^{5+} \mathrm{BIM}$ in terms of $V_{p} / V_{\text {the }} n_{e}=10^{25} \mathrm{e} \mathrm{cm}^{-3}$. Alpha denotes proton concentration

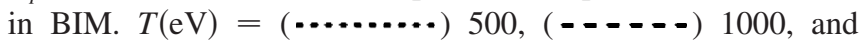
( - - - ) 2000. Dark gray (red) pertains to $e$ stopping, light gray (green) to $\mathrm{H}^{+}$stopping, and black (blue) to $\mathrm{B}^{5+}$ stopping.

FIG. 5. (Color) Proton stopping in $\mathrm{H}^{+}-\mathrm{U}_{238}^{+}$BIM in terms of $V_{p} / V_{\text {the }} \cdot n_{e}=10^{23} \mathrm{e} \mathrm{cm}^{-3}$. Alpha denotes proton concentration

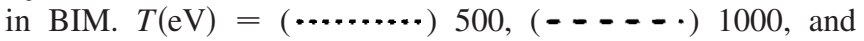
( - - - ) 5000. Dark gray (red) pertains to $e$ stopping, light gray (green) to $\mathrm{H}^{+}$stopping, and black (blue) to $\mathrm{H}^{+}$stopping. $\mathrm{U}_{238}^{+}$stopping.

Then, the $\mathrm{U}_{238}^{+}$(blue stopping contribution crosses the $\mathrm{H}^{+}$ green) at $V_{p} / V_{\text {the }} \leq 0.01$.

Such a large $M_{2} / M_{1}$ ratio induces a strong localization of the heaviest ion stopping efficiency very close to very small ion projectile $V_{p}$ values. Figures 4 and 5 clearly demonstrate that as soon as $\alpha \geq 0,5$, the heaviest $\mathrm{U}_{238}^{+}$ stopping contribution can be quantitatively neglected in front of the proton one. The latter (Fig. 5) extends over the full $V_{p}$ range, up to a crossing area with electron linear curves. This phenomenon highlights a much easier energy transfer between incoming proton projectile and target protons than with the hardly mobile $\mathrm{U}_{238}^{+}$ions. Very similar behaviors are featured by the same BIM immersed in much more dilute plasmas of magnetic fusion energy (MFE) concern with $n_{e}=10^{14} \mathrm{e}-\mathrm{cm}^{-3}$.

\section{CHARGE ASYMMETRY}

\section{A. Proton-boron $11\left(Z_{2} / Z_{1}=5, M_{2} / M_{1}=10.81\right)$ BIM}

Switching interest to BIM with asymmetric charge distribution, we first pay attention to a specific fuel of alter-

native thermonuclear fusion significance [9] yielding no neutron output, and immersed in a dense plasma surrounding with $n_{e}=10^{25} \mathrm{e}-\mathrm{cm}^{-3}$ and $T(\mathrm{eV})=500,1000$, and 2000. An immediate inspection of Fig. 6 demonstrates that the highest charge and heavies $\mathrm{B}_{11}^{5+}$ BIM component provides, at all $\alpha$ values, the overwhelming stopping contribution when the target is submitted to low velocity proton beams. So, in the present situation, the target proton LVISD may be safely neglected when contrasted to that of $\mathrm{B}_{11}^{5+}$ and the electrons.

\section{B. Astrophysical BIM}

We now turn attention to two BIM of very important astrophysical pertinence, $\mathrm{He}^{2+}{ }_{-} \mathrm{C}^{6+}$ featuring white dwarfs (WD) crust [10] and $\mathrm{Si}^{14+}-\mathrm{Sn}^{36+}$ relevant to ion interdiffusion issues in stellar envelopes [11]. Both BIM feature a charge ratio $2.5 \leq Z_{2} / Z_{1} \leq 3$ and mass ratio $M_{2} / M_{1} \sim 3$, in dense plasmas with IFE-like parameters. Considering first, $\mathrm{He}^{2+}-\mathrm{C}^{6+}$, associated to the "carbon pollution" issue in WD, one has $n_{e}=10^{26} \mathrm{e}-\mathrm{cm}^{-3}$ with $T(\mathrm{keV})=1,2$, and 5 (see Fig. 7), which as the above proton-, $\mathrm{B}_{11}^{5+}$ BIM advocates that $\mathrm{C}^{6+}$ dominates LVISD, at any relative proportion for $T \leq 2 \mathrm{keV}$, when the target is exposed to incoming protons. In Fig. 7, $V_{p}$ is measured in $V_{\text {th1 }}$ for $\alpha \leq$ 0.5 , and in $V_{\text {th2 }}$ for $\alpha \geq 0.5$. Both scalings are compared in 

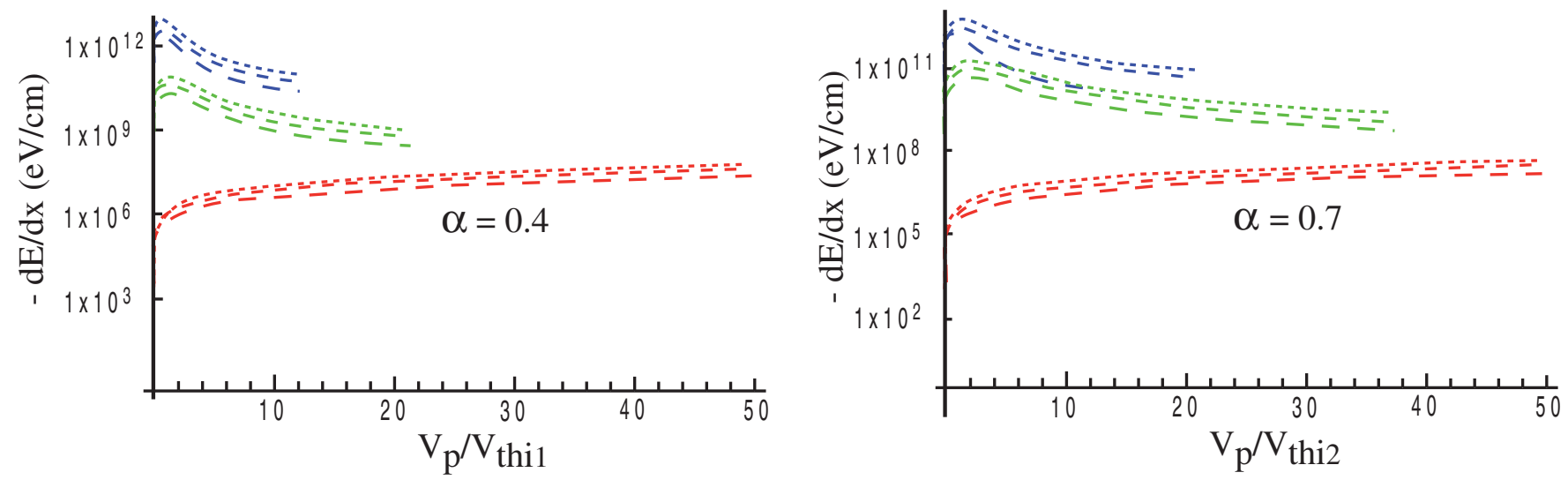

FIG. 7. (Color) Proton $-d E / d x(\mathrm{eV} / \mathrm{cm})$ with respect to $V_{p} / V_{\text {thi }}$ in a $\mathrm{He}^{2+}-\mathrm{C}^{6+} \mathrm{BIM}$ at $n_{e}=10^{26} \mathrm{e} \mathrm{cm}^{-3}$ with $T(\mathrm{keV})=(\cdots \cdots \cdots) 1$, $(-\ldots) 2$, and $(---\cdot)$ 5. $\alpha=\mathrm{He}^{2+}$ concentration. Dark gray (red) denotes $e$ stopping, light gray (green) pertains to $\mathrm{He}^{2+}$ stopping, and black (blue) to $\mathrm{C}^{6+}$ stopping.

Fig. 8 for $\alpha=0.5$. As above, the electron LVISD does not show up on these very low velocity linear scales, while the $\mathrm{He}^{2+}$ contribution hardly appears when $V_{p}$ is scaled by $V_{\text {thi2 }}$.

Turning to $\mathrm{Si}^{14+}-\mathrm{Sn}^{36}$ BIM immersed also in a dense plasma impacting $\alpha$ particles and advocated $n_{e}\left(\mathrm{~cm}^{-3}\right)=$ $10^{26}$ and $T(\mathrm{keV})=100$ and 200, with pertaining to LVISD pictured on Figs. 9 with linear $-d E / d x(\mathrm{eV} / \mathrm{cm})$. Within
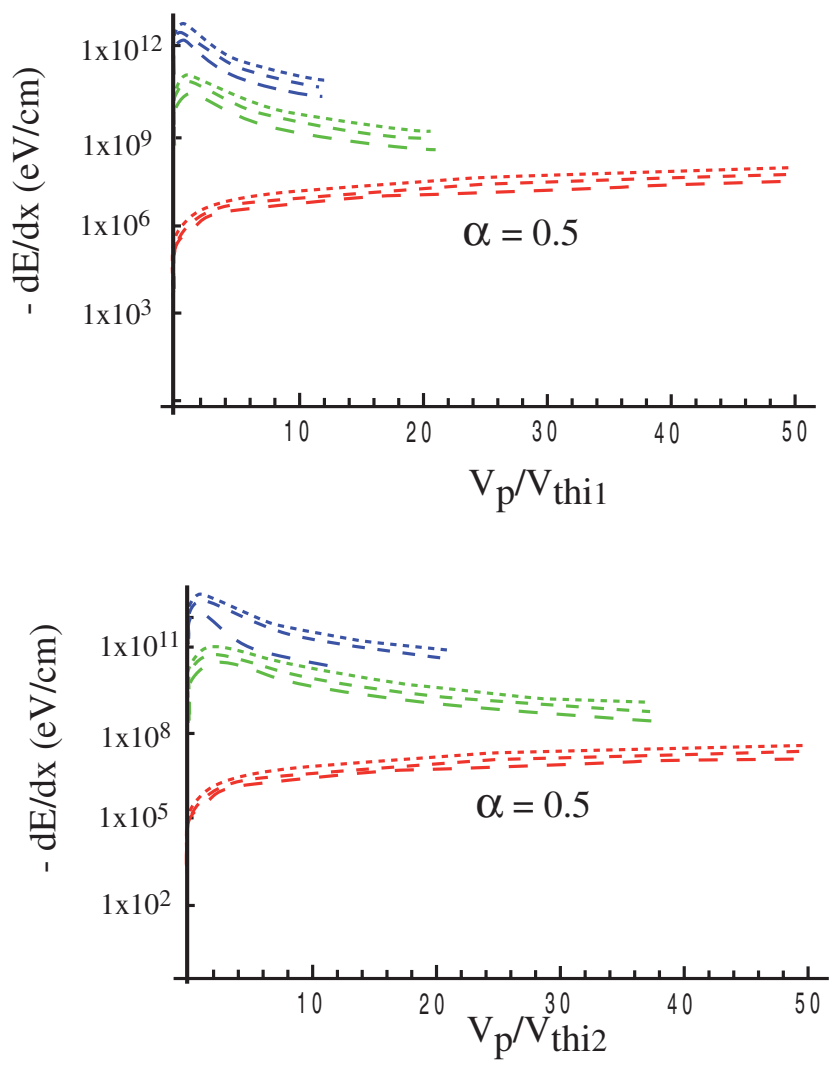

the very low $V_{p} / V_{\text {thi }}$ considered range, electron contribution has vanished and the $\mathrm{Si}^{14}$ one should be quantitatively included only for $\alpha=0.9$ and $V_{p} / V_{\text {th2 }} \geq 4$. It should be recalled that in Fig. 9 as well as in many previous ones, we intend to contrast LVISD through the $\alpha \Leftrightarrow 1-\alpha$ exchange, and so depart to a certain extent from the usual increasing $\alpha$ monotone presentation.
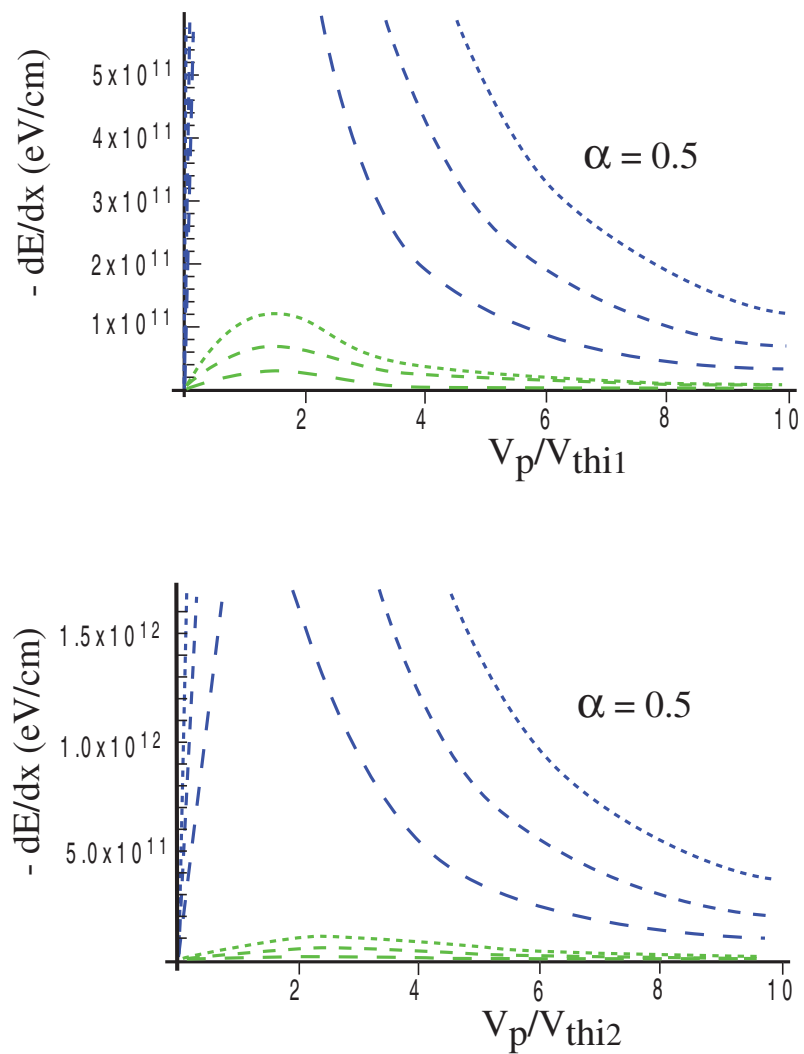

FIG. 8. (Color) Proton $-d E / d x(\mathrm{eV} / \mathrm{cm})$ with respect to $V_{p} / V_{\text {thi }}$ in a $\mathrm{He}^{2+}{ }_{-} \mathrm{C}^{6+} \mathrm{BIM}$ at $n_{e}=10^{26} \mathrm{e} \mathrm{cm}^{-3}$ with $T(\mathrm{keV})=$ (............) 1 , (- - - - . 2 ( - - - 5. $5=\mathrm{He}^{2+}$ concentration. Dark gray (red) denotes $e$ stopping, light gray (green) pertains to $\mathrm{He}^{2+}$ stopping, and black (blue) to $\mathrm{C}^{6+}$ stopping. 

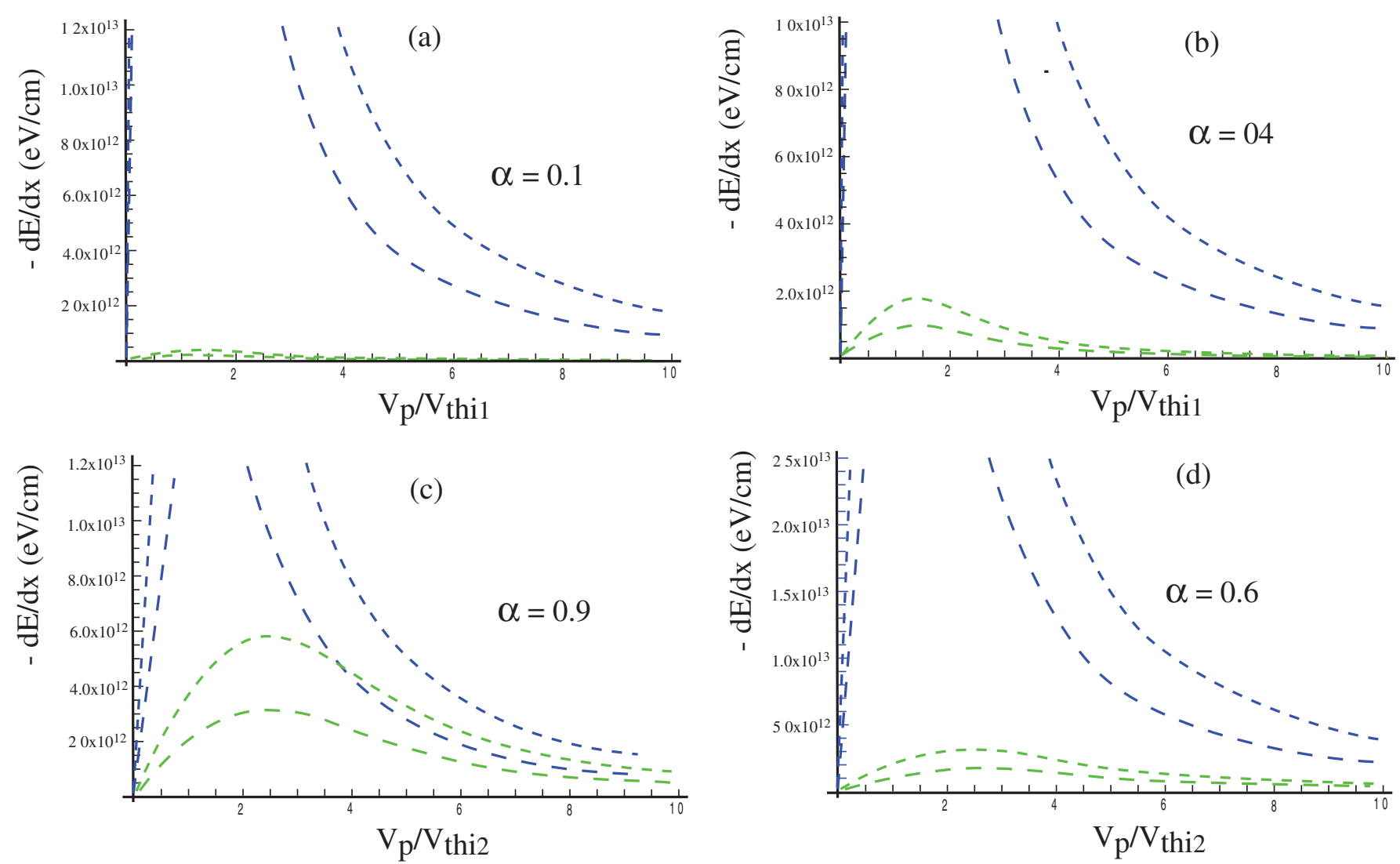

FIG. 9. (Color) Alpha particle projectile in a $\mathrm{Si}^{14+}-\mathrm{Sr}^{36+} \mathrm{BIM}$ with a $n_{e}=10^{26} \mathrm{e} \mathrm{cm}^{-3}$ and temperatures $=(-----\cdot) 100 \mathrm{keV}$, ( - - - ) $200 \mathrm{keV}$ with $\mathrm{Si}^{14+}$ relative proportion: (a) $\alpha=0.1$, (b) $\alpha=0.4$, (c) $\alpha=0.9$, and (d) $\alpha=0.6$. Dark gray (red) refers to electron stopping, light gray (green) to $D^{+}$stopping, and black (blue) denotes $T^{+}$stopping, $-d E / d x(\mathrm{eV} / \mathrm{cm})$ in terms of $V_{p} / V_{\text {thi }}$.

\section{PBAR- $D^{+}\left(Z_{2} / Z_{1}=-1, M_{2} / M_{1}=2\right)$}

If one pushes the BIM charge dissymmetry up to including negatively charged ions, one reaches novel LVISD behaviors as displayed in Fig. 4 by the antiproton (pbar)- $\mathrm{D}^{+}$mixture submitted to $\mathrm{He}^{2+}$ projectiles. In contradiction to the previous patterns (Figs. 6-9), one now witnesses a rather well approximated quasisymmetry between $\alpha=0.4$ and $\alpha=0.6$ (see Fig. 10). The conspicuous feature now arises at $\alpha=0.5$, when the two target ion LVISD exactly compensate each other, leaving only a much reduced electron stopping, within the given overall charge neutral target BIM.

The slight remaining asymmetric discrepancy arises from the mass ratio $M_{2} / M_{1}=2$ rendering $D^{+}$a little less mobile than the pbar in a dense and hot plasma $\left[n_{e}=\right.$ $10^{23} \mathrm{e}-\mathrm{cm}^{-3}$ with $T(\mathrm{keV})=0.1$ and 1]. So the $\alpha$-pbar attraction results in a noticeable larger LVISD at $\alpha=0.6$ compared to that for deuton stopping at $\alpha=0.4$.

\section{SUMMARIES AND OUTLOOK}

As a first conclusive remark, we can notice that Eq. (11) usually fits rather well the linearly rising part of the LVISD curves.
The rather detailed exploration of low velocity ion stopping in several BIM of potentially high interest in thermonuclear fusion and astrophysics should allow a novel reinvestigation of the nuclear reaction rates at work in these systems. Moreover, the present dielectric formulation albeit restricted to a dense and classical electron fluid, could be easily extended to partially Fermi degenerate situations of obvious practical interest, by introducing the corresponding $\hbar \neq 0$ Fried-Conte extension [12,13].

In this regard, the matching of LVISD in these targets with an estimate of low ion velocity multiple scattering would also be of high interest [14].

In these regards, the ion projectile scattering probability writes as [14]

$$
\frac{d P}{d q_{\perp}}=V_{p} \times G_{\forall T}\left(q_{\perp}\right),
$$

where

$$
\begin{aligned}
G_{\forall T}\left(q_{\perp}\right)= & \frac{4 Z^{2} e^{4} m_{e}^{2}}{\hbar^{4} \pi} q_{\perp} \int_{q_{\perp}}^{\infty} \frac{1}{\left(q^{2}+q_{s}^{2}\right)^{2}} \\
& \times \frac{1}{1+\exp \left(\frac{\hbar^{2}}{8 m_{e} T} q^{2}-\eta\right)} d q
\end{aligned}
$$




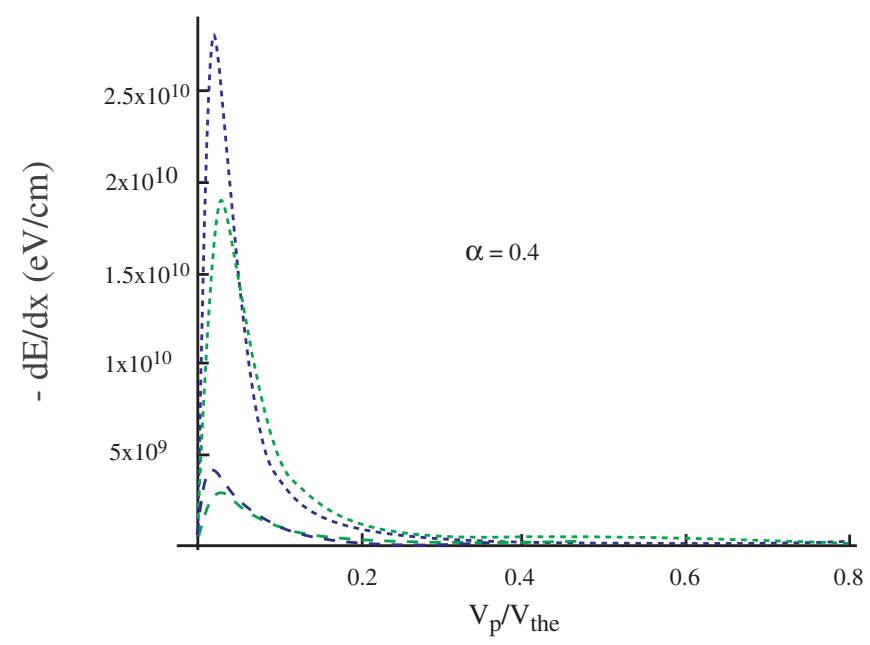

Equation (12) advocates the $V_{p}$-linear dependence already featuring LVISD. However, the projectile-target mechanisms are markedly distinct, LVISD is monitored by target ion stopping, while present multiple scattering is essentially operated by target electrons.

Finally, inclusion of dynamical intrabeam correlations should document a realistic ion beam-plasma interaction at low velocity [15].

Starting with the charge quasisymmetric DT BIM, one perceives the charge asymmetry as an organizing concept in the data analysis.

\section{ACKNOWLEDGMENTS}

This work has been performed under the auspices of

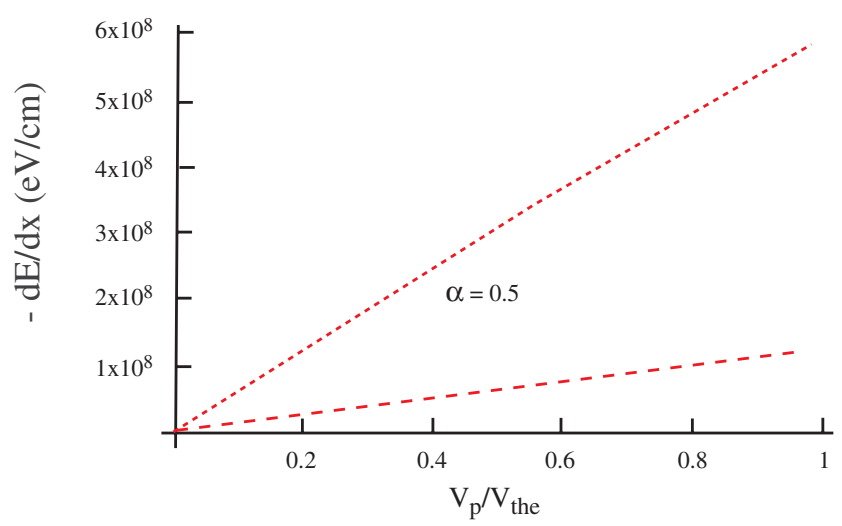
CEA/EURATOM Contract No. V35599.001 for which the authors are deeply grateful.

[1] P. Sigmund and A. Schinner, Nucl. Instrum. Methods Phys. Res., Sect. B 195, 64 (2002).

[2] G. Logan, F. Bieniosek, C. Celata, E. Henestroza, J. Kwan, E. P. Lee, M. Leitner, L. Prost, P. Roy, P. A. Seidl, S. Eylon, J. L. Vay, W. Aldron, S. Hu, J. Barbard, D. Callahan, R. Cohen, A. Friedman, D. Grote, M. K. Covo, W. R. Meier, A. Molvik, S. Lund, R. Davidson, P. Efthimion, E. Gilson, L. Grisham, I. Kaganovich, H. Qin, E. Startsev, D. Rose, D. Welch, C. Olson, R. Kishek, P. O'Shea, and I. Haber, Nucl. Instrum. Methods Phys. Res., Sect. A 544, 1 (2005).

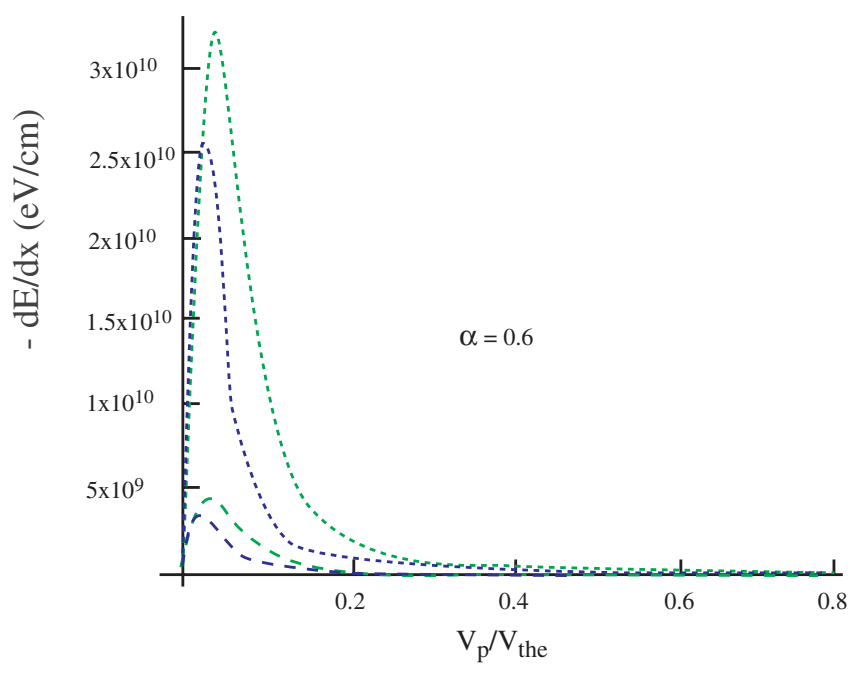

FIG. 10. (Color) $\mathrm{He}^{2+}-d E / d x(\mathrm{eV} / \mathrm{cm})$ in terms of $V_{p} / V_{\text {the }}$ in a pbar- $D^{+}$BIM with total ion density $=10^{23} \mathrm{e} \mathrm{cm}^{-3} . \alpha$ denotes relative pbar concentration. Dark gray (red) refers to $e$ stopping light gray (green) to pbar stopping, and black (blue) $\mathrm{H}^{+}$stopping. $\mathrm{TeV}=(\ldots \ldots \ldots . . . .)$.100 , and (- - - - - $) 1000$.

with $q_{\perp}$, transverse projectile (charge Z) momentum, while $q_{s}^{2}$ qualifies target electron density and temperature with the usual $\eta=\beta_{\mu}$ normalized chemical potential.

[3] L. S. Grisham, Phys. Plasmas 11, 5727 (2004).

[4] G. Zwicknagel, C. Toepffer, and P.-G. Reinard, Phys. Rep. 309, 117 (1999).

[5] B.D. Fried and S.D. Conte, The Plasma Dispersion Function (Academic Press, New York, 1961).

[6] C. Deutsch, Ann. Phys. (Paris) 11, 1 (1986); C. Deutsch and G. Maynard, Recent Res. Dev. Plasmas 1, 1 (2000).

[7] Th. Peter and J. Meyer-ter-Vehn, Phys. Rev. A 43, 1998 (1991).

[8] M. Tabak, J. Hammer, M. E. Glinsky, W. L. Kruer, S. C. Wilks, J. Woodworth, E. M. Campbell, M. D. Perry, and R. J. Mason, Phys. Plasmas 1, 1626 (1994); C. Deutsch, Eur. Phys. J. Appl. Phys. 24, 95 (2003).

[9] D. C. Moreau, Nucl. Fusion 17, 13 (1977); S. Eliezer and J. M. Martinez-Val, Laser Part. Beams 16, 581 (1998).

[10] See, for instance, L. Siess, Astron. Astrophys. 448, 717 (2006).

[11] D. B. Boercker and E. L. Pollock, Phys. Rev. A 36, 1779 (1987).

[12] C. Deutsch, Phys. Lett. 60A, 317 (1977); N. R. Arista and W. Brandt, Phys. Rev. A 29, 1471 (1984).

[13] C. Gouedard and C. Deutsch, J. Math. Phys. (N.Y.) 19, 32 (1978); R. G. Dandrea, N. W. Ascroft, and A. E. Carlsson, Phys. Rev. B 34, 2097 (1986).

[14] R. Popoff, G. Maynard, and C. Deutsch, Phys. Rev. E 80, 046408 (2009).

[15] C. Deutsch and N. A. Tahir, Phys. Fluids B 4, 3735 (1992). 